Notfall Rettungsmed 2022 25 (Suppl 2):S39 https://doi.org/10.1007/s10049-021-00972-z Online publiziert: 12. Januar 2022

(c) The Author(s), under exclusive licence to Springer Medizin Verlag GmbH, ein Teil von Springer Nature 2022

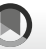

\section{Erratum zu: „D-Probleme“ des nichttraumatologischen Schockraummanagements}

Mark Michael' $\cdot$ Bernhard Kumle ${ }^{2}$ Martin Pin ${ }^{3}$. Niels Hammer ${ }^{4,5,6} \cdot$ Michael Gliem $^{7}$. Adisa Kuršumović ${ }^{8} \cdot$ Michael Bernhard'

'Zentrale Notaufnahme, Universitätsklinikum Düsseldorf, Heinrich-Heine-Universität, Düsseldorf, Deutschland; ${ }^{2}$ Klinik für Akut- und Notfallmedizin, Schwarzwald-Baar-Klinikum, Villingen-

Schwenningen, Deutschland; ${ }^{3}$ Zentrale Notaufnahme, Florence-Nightingale-Krankenhaus, Düsseldorf, Deutschland; ${ }^{4}$ Institut für Klinische und Makroskopische Anatomie, Medizinische Universität Graz, Graz, Österreich; ${ }^{5}$ Klinik für Orthopädie und Unfallchirurgie, Universität Leipzig, Leipzig, Deutschland;

${ }^{6}$ Abteilung Medizintechnik, Fraunhofer Institut für Werkstoff- und Umformtechnik, Dresden, Deutschland; ${ }^{7}$ Klinik für Neurologie, Universitätsklinikum Düsseldorf, Heinrich-Heine-Universität, Düsseldorf, Deutschland; ${ }^{8}$ Klinik für Neurochirurgie, Sektion Interventionelle Neuroradiologie, Schwarzwald-BaarKlinikum, Villingen-Schwenningen, Deutschland

\section{Erratum zu:}

Notfall Rettungsmed 2021

https://doi.org/10.1007/s10049-021-

00915-8

Im ursprünglich publizierten Beitrag ist in - Abb. 1 die Beschriftung der dargestellten zerebralen Blutungen nicht korrekt zugeordnet.

Bitte beachten Sie die hier korrigierte Darstellung: Abb. 1a subdurale Blutung und Abb. $1 \mathrm{~b}$ epidurale Blutung.

Wir bitten den Fehler zu entschuldigen. Der Originalbeitrag wurde korrigiert.

\section{Korrespondenzadresse}

Prof. Dr. med. Michael Bernhard, MHBA

Zentrale Notaufnahme, Universitätsklinikum Düsseldorf, Heinrich-Heine-Universität Moorenstraße 5, 40225 Düsseldorf, Deutschland

michael.bernhard@med.uni-duesseldorf.de

Die Online-Version des Originalartikels ist unter https://doi.org/10.1007/s10049-021-00915-8 zufinden.

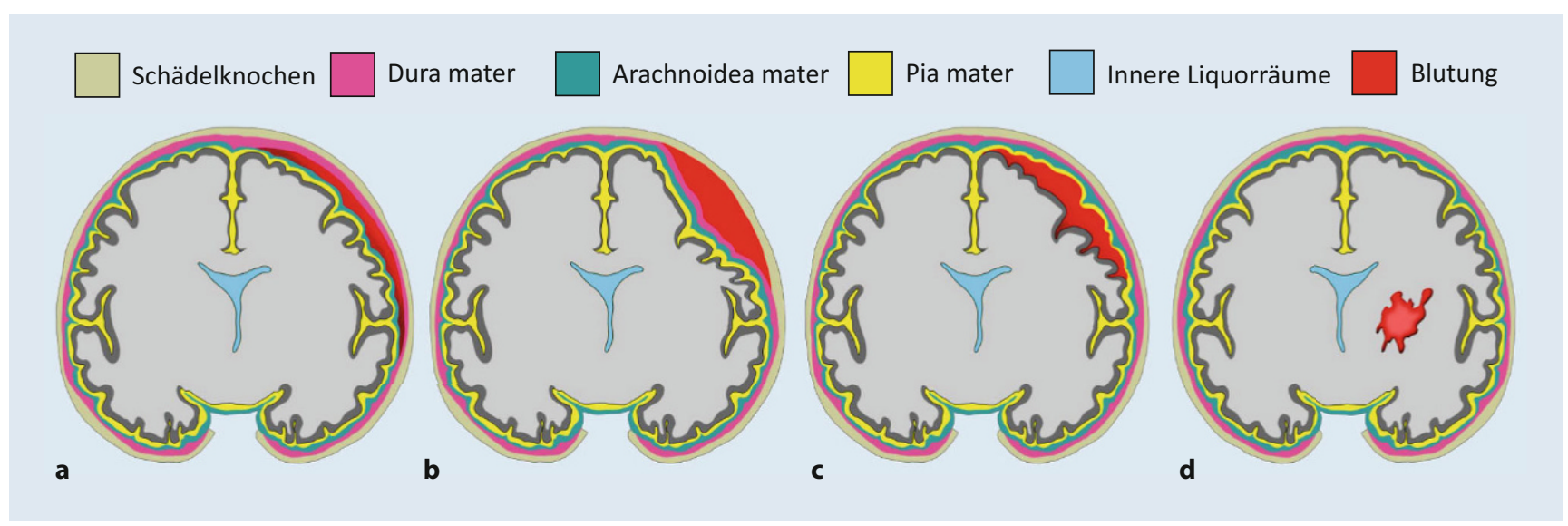

Abb. 1 ム Zerebrale Blutungen: a subdurale Blutung, b epidurale Blutung, c subarachnoidale Blutung, d intrazerebrale Blutung. Es handelt sich um eine vereinfachte Darstellung in der Koronarebene. Blutungsherde sind rot dargestellt, deren Lage relativ zu den Hirnhäuten beschreibt die Blutungsform. (Mit freundl. Genehmigung, @ Prof. Dr. med. Niels Hammer, alle Rechte vorbehalten) 